\title{
Über die chemische Zusammensetzung des roten Salztones.
}

\author{
Nach Analysen von E. Marcus, \\ mitgeteilt von Wilheli Biltz.
}

Vor einiger Zeit berichteten wir an dieser Stelle über vollständige Analysen von grauem Salzton aus StaBfurt. ${ }^{1}$ AuBer dem grauen Ton findet sich in der geologischen Literatur der Salzlagerstätten eine andere Tonvarietät als „roter Salzton“ angeführt, die inmitten des jüngeren Steinsalzlagers auftritt und zuerst von ZIM.MERMANN ${ }^{2}$ beschrieben worden ist. In diese Schicht rechnet man auch Anteile, die den Namen insofern nicht rechtfertigen, als sie blaugrünlich gefärbt sind. Doch treten die blaugrünen mit den roten Partien in unmittelbarer Nachbarschaft und ineinander verwachsen auf und der Unterschied der Farbe macht, wie auch hier gezeigt werden wird, keinen Wesensunterschied aus. Der rote Ton ist in jungster Zeit von ReIDemeister ${ }^{3}$ auf seine Hauptbestandteile hin analysiert worden. Präzisionsanalysen fehlen indessen und zur Abrundung der früheren Arbeit mögen daher die nachfolgenden Angaben über vollständige Analysen von Proben aus dieser Gebirgsschicht Platz finden.

Die drei ersten Proben entstanimen einer typischen Schicht des roten Tones aus dem jüngeren Steinsalzlager zu Schönebeck; sie sind aus der Firste der südwestlichen Hauptstrecke der $414.5 \mathrm{~m}$ Sohle des Graf Moltke-Schachtes zu Schönebeck entnommen. Die Schichtenfolge rom Liegenden zum Hangenden ist dort: Jüngeres, stark rötlich gefärbtes Steinsalz; blaugrüner Ton (Probe 1); roter und blaugrüner Ton gemengt (Probe 2; doch wurde der blaugrüne Ton möglichst ausgespart); roter Ton (Probe 3); Pegmatitanhydrid;

1 Z. anorg. Chem. 68 (1910), 91.

2 Zeitschr. deutsch. Geol. Ges. 59 (1907), 136; Zeitschr. Kali 3 (1909), 14.

${ }^{3}$ Dissertation, Kiel 1911. 
jüngeres Steinsalz hell rötlich. Die Mächtigkeiten in der Schicht betragen etwa: blaugrüner gemengter Ton $1.80 \mathrm{~m}$; roter Ton $3.20 \mathrm{~m}$; Pegmatitanhydrit $1 \mathrm{~m}$. Durch Faltung sind die Schichten derart aufgerichtet, daß sie bei der Horizontalbefahrung durchquert werden. Im Hangenden des jüngeren Steinsalzes trifft man in Schönebeck auf die Buntsandsteintondecke, die das gesamte Salzlager abschlieBt. Aus dem Kontakt dieses Gesteins mit dem Salz wurde Probe 4 entnommen. Dem Kgl. Salzamte Schönebeck sind wir für die Unterstützung bei der Probenahme zu lebhaftem Danke verbunden. Eine fünfte, rein rote Tonprobe stammte aus dem jüngeren Steinsalz von Sperenberg, Bohrloch X, wahrscheinlich aus 707-711 m Tiefe. Wir wählten dieses Beispiel aus einer Sendung aus, die wir der besonderen Liebenswürdigkeit Herrn Prof. Zimmermanns verdanken.

Über die Vorbereitung der Proben und ihre Analyse ist das Nähere bereits in der erwähnten Arbeit über den StaBfurter Ton mitgeteilt. Bei der Bestimmung der Hauptbestandteile ist hier noch anzuführen, $\mathrm{da} B \quad 0.03-0.10 \%$ Kieselsäure mit in den wasserlöslichen Teil übergingen; dieser Anteil erscheint in der Zusammenstellung der Ergebnisse nicht gesondert, sondern es wird dort nur die bei der Hauptanalyse insgesamt gefundene Kieselsäure angeführt. Die Fällung der alkalischen Erden kann ohne vorherige Zerstörung in der Lösung enthaltenen Ammoniumsultids erfolgen, wie wir übereinstimmend mit HrLlezBrand stets bestätigen konnten. Von den untergeordneten Bestandteilen bestimmten wir, wie früher $\mathrm{TiO}_{2}$, $\mathrm{P}_{2} \mathrm{O}_{5}, \mathrm{Vd}_{2} \mathrm{O}_{3}$ und $\mathrm{C}$. Eine besondere Prüfung auf Bitumen fand nicht statt; ebensowenig auf Zirkon und seltene Erden, da eine solche bei den früheren Tonen stets ergebnislos gewesen war. In dem Sperenberger Ton konnte Borsäure quantitativ bestimmt werden. Qualitativ wurde überall auf sulfidischen Schwefel, auf $\mathrm{Ni}, \mathrm{Cr}, \mathrm{Li}$, $\mathrm{Rb}, \mathrm{Br}$ und $\mathrm{B}_{2} \mathrm{O}_{3}$ geprüft. In einem Falle zeigte sich eine Spur Kupfer. Auf eine Trennung von $\mathrm{Mn}$ und $\mathrm{Zn}$ wurde verzichtet. Keiner der untersuchten Tone enthält Eisen in wasserlöslicher Form.

Zur Berechnung verfuhr man ganz, wie früher, in doppelter Weise. In der ersten Tabelle sind die wasserlöslichen Anteile nach Ionen berechnet angegeben und die des wasserlöslichen Teiles so, wie dies in der Silikatanalyse üblich ist. In der zweiten Tabelle werden die negativen und positiven Bestandteile des wasserlöslichen Teiles willkürlich aufeinander bezogen. Das hat hier keine andere sachliche Begründung, als daß man sieht, ob sie sich stöchiometrisch 


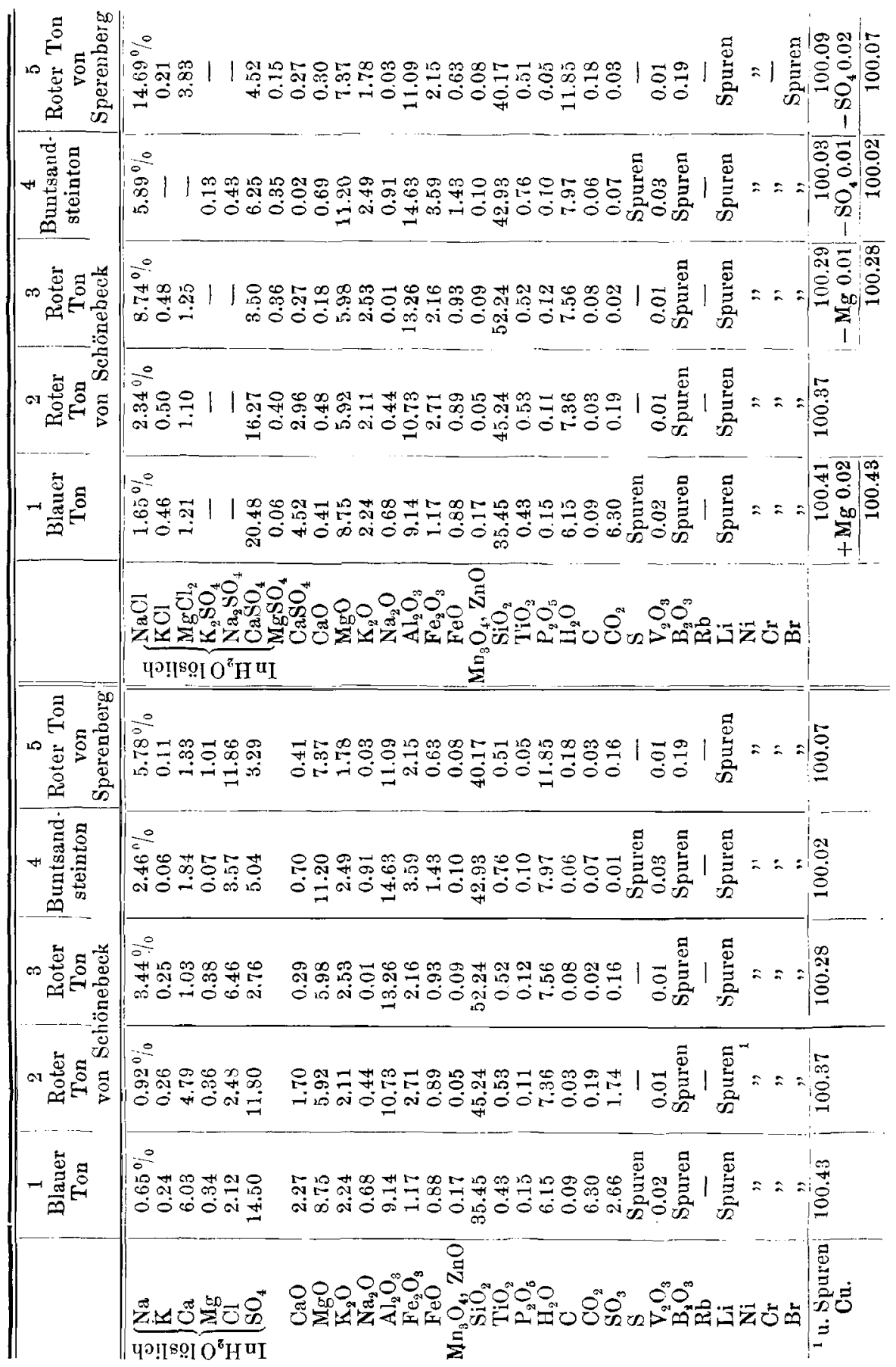


einander ergänzen. Es ist dies innerhalb $0.02 \%$ Differenz zwischen dem Sollwert und dem gefundenen Wert der Fall, um welchen Betrag am SchluB die Summe aller Bestandteile zu korrigieren ist. Die Summe der gefundenen Prozente fällt mit 100.02-100.43\% in die nach HILLEBrand für vollständige Analysen zulässigen Grenzen.

Grundsätzliche Unterschiede zwischen den Tonen bestehen weder in den gewöhnlichen, noch in den untergeordneten Bestandteilen. Vergleicht man die drei ersten, an benachbarten Stellen genommenen Proben, so sieht man, wie rom Liegenden zum Hangenden der $\mathrm{CaSO}_{4}$ - und $\mathrm{CO}_{2}$-Gehalt fällt, der $\mathrm{Al}_{2} \mathrm{O}_{3}$ - und $\mathrm{SiO}_{2}$-Gehalt steigt. Das Eisenoxyd waltet in den roten Tonen gegenüber dem Oxydul stark vor; in dem blaugrünen Ton tritt es zurück, wodurch sich dessen Farbe erklärt. Von den Staßfurter Proben waren Tone im eigentlichen Sinne des Wortes nur die Mittellagen, während das Liegende stark polyhalithaltig, das Hangende stark magnesithaltig war. Von dem chemischen Charakter der tonigen Mittelschicht weicht der der roten Tone nur unbedeutend ab. Die vierte Probe, der Bundsandsteinton aus dem Salzkontakt, weist gegenüber dem roten Salzton ebenfalls keine auffallenden Unterschiede auf und ebensowenig der Ton aus Sperenberg, sofern man nicht in dem hohen Wasser- und in dem Borsäuregehalt einen solchen erblicken will. $\mathrm{Zu}$ irgendwelcher Besonderheit chemischer Art hat die Analyse dieser Gesteine somit nicht geführt.

Die von REIDEMEISTER angeführten Teilanalysen der grauen und roten Tone decken sich im allgemeinen mit unseren vollständigen Analysen, soweit die Verschiedenartigkeit der Probenahme und der Arbeitsweise dies erwarten läßt. Die Alkalimetalle werden von REIDEMEISTER entweder als Chloride in Rechnung gesetzt, ohne daB eine Chlorbestimmung ausgeführt wurde, oder überhaupt nicht bestimmt. Das erste Verfahren ist ziemlich willkürlich und würde z. B. bei unserem Stabfurter Ton Nr. 21 und bei den Schönebecker Tonen zu mehr als $100 \%$ führen, da die Alkalimetalle in Wirklichkeit zum Teil auch als Sulfate und als oxydische Bestandteile des Wasserunlöslichen vorliegen. Ein Vergleich ergibt, daß bei den Stabfurter Tonen unsere Analyse Nr. 22 etwa der Nr. 1 von ReIDEmeister entspricht, Nr. 23 von uns der Nr. 3 von Reidemeister; und die Probe 6 von ReIDemerster liegt etwa zwischen Nr. 23 und Nr. 24 von unserer Serie. Die Diagramme Reidemeisters über den Kaolin-, den Kieselsäure-, den Eisenoxyd- und den Eisenoxydul- 
E. Marcus u. W. Biltr. Chemische Zusammensetzung d. roten Salztones. 123 gehalt der Tone können ohne wesentliche Abweichung auch unter Zugrundelegung unserer Zahlen gezeichnet werden.

Bei den roten und blaugrünen Schönebecker Tonen tritt der Unterschied im Eisenoxydgehalte bei REIDEMEISTER etwas deutlicher zutage als hier. Der Oxydgehalt der blaugrünen Probe liegt unter dem Oxydulgehalt, der der roten, wie bei uns, darüber.

Dem Verbande zur wissenschaftlichen Erforschung der deutschen Kalisalzlagerstätten sind wir für weitere Bewilligung von Geldmitteln für diese Fortsetzung unserer Arbeiten dankbar.

Clausthal i. H., Kgl. Betriebslaboratorium.

Bei der Redaktion eingegangen am 25. Mai 1912. 